\title{
A NOTE ON THE CLOSURE OF CONVOLUTION POWER MIXTURES (RANDOM SUMS) OF EXPONENTIAL DISTRIBUTIONS
}

\author{
J. M. P. ALBIN
}

\author{
(Received 11 April 2007; revised 21 July 2007)
}

Communicated by V. T. Stefanov

\begin{abstract}
We make a correction to an important result by Cline [D. B. H. Cline, 'Convolutions of distributions with exponential tails', J. Austral. Math. Soc. (Series A) 43 (1987), 347-365; D. B. H. Cline, 'Convolutions of distributions with exponential tails: corrigendum', J. Austral. Math. Soc. (Series A) 48 (1990), 152-153] on the closure of the exponential class $\mathcal{L}(\alpha)$ under convolution power mixtures (random summation).
\end{abstract}

2000 Mathematics subject classification: primary 60F99, 62E20; secondary 44A10, 60E07.

Keywords and phrases: compound Poisson distribution, convolution power, exponential distribution, random sum, Tauberian theorem.

\section{Introduction}

We say that a cumulative probability distribution function $H$ on the real line belongs to the exponential class $\mathcal{L}(\alpha), \alpha>0$, if

$$
\lim _{x \rightarrow \infty} \frac{\bar{H}(x+t)}{\bar{H}(x)}=\lim _{x \rightarrow \infty} \frac{1-H(x+t)}{1-H(x)}=e^{-\alpha t} \quad \text { for } t \in \mathbb{R} .
$$

(Some authors require members of $\mathcal{L}(\alpha)$ to be supported on $[0, \infty)$ to avoid some technicalities. However, because of the applications we have in mind (see, for example, Section 4 below) we do not want to make this restriction.) Note the elementary fact that (1) holds for $t \in \mathbb{R}$ if and only if it holds for $t>0$. Furthermore, $H \in \mathcal{L}(\alpha)$ if and only if $H \circ \log$ is regularly varying at infinity with index $-\alpha$.

When (1) holds with $\alpha=0$ we get the class of long-tailed distributions $\mathcal{L}(0)=\mathcal{L}$ introduced by Pitman [10]. Note the elementary fact that $H \in \mathcal{L}$ if and only if

(C) 2008 Australian Mathematical Society 1446-7887/08 \$A2.00+0.00 


$$
\limsup _{x \rightarrow \infty} \frac{\bar{H}(x-t)}{\bar{H}(x)} \leq 1 \quad \text { or } \quad \liminf _{x \rightarrow \infty} \frac{\bar{H}(x+t)}{\bar{H}(x)} \geq 1 \quad \text { for some } t>0 .
$$

Furthermore, $H \in \mathcal{L}$ if and only if $H \circ \log$ is slowly varying at infinity.

The class $\mathcal{L}(\alpha)$ was introduced by Embrechts and Goldie [6] to study closedness properties of the narrower exponential class $\mathcal{S}(\alpha)$ consisting of those $H \in \mathcal{L}(\alpha)$ for which

$$
\lim _{x \rightarrow \infty} \frac{\overline{H^{\star 2}}(x)}{\bar{H}(x)}=\lim _{x \rightarrow \infty} \frac{\overline{H \star H}(x)}{\bar{H}(x)}=\lim _{x \rightarrow \infty} \frac{1-\int_{\mathbb{R}} H(x-y) d H(y)}{1-H(x)}
$$

exists (and is finite). Members of the class $\mathcal{S}(0)=\mathcal{S}$ are called subexponential distributions. The classes $\mathcal{S}(\alpha), \alpha \geq 0$, have a much longer history than $\mathcal{L}(\alpha)$; see, for example, Cline [4] for more information.

In an important paper Cline [4] established many important results for the classes $\mathcal{L}(\alpha)$ and $\mathcal{S}(\alpha)$. It has recently been pointed out by Shimura and Watanabe [13, Remark 4.2], that Cline [4, Lemma 2.1(iv)] is incorrect, which has some consequences for later results that build on that lemma.

It was noted by Cline himself in [5] that there is also a problem with Cline [4, Lemma 2.3(ii)], which claims that, given a cumulative probability distribution function $F$ supported on $[0, \infty)$ (which is to say that $F(x)=0$ for $x<0$ ), together with discrete probabilities $\left\{\lambda_{n}\right\}_{n=0}^{\infty}$ such that $\lambda_{0}<1$ and $\sum_{n=0}^{\infty} \lambda_{n}=1$, the convolution power mixture (random sum distribution) $H(x)=\sum_{n=0}^{\infty} \lambda_{n} F^{\star n}(x)$ satisfies the following implication

$$
\text { if } F \in \mathcal{L}(\alpha) \text { then } \liminf _{x \rightarrow \infty} \frac{\bar{H}(x+t)}{\bar{H}(x)} \geq e^{-\alpha t} \text { for } t>0 \text {, for } \alpha \geq 0 .
$$

In particular, (2) together with (4) show that $F \in \mathcal{L}$ implies $H \in \mathcal{L}$.

In Cline [5] the result (4) is not only corrected, but also strengthened to the result that $F \in \mathcal{L}(\alpha)$ implies $H \in \mathcal{L}(\alpha)$ for $\alpha \geq 0$. However, we have the following simple counterexample to the claimed result of Cline [5] for $\alpha>0$.

Proposition 1.1. Given constants $0<\gamma<\alpha<\infty$ and an $F \in \mathcal{L}(\alpha)$ that is supported on $[0, \infty)$, there exist probabilities $\left\{\lambda_{n}\right\}_{n=0}^{\infty}$ such that $H=\sum_{n=0}^{\infty} \lambda_{n} F^{\star n}$ $\notin \mathcal{L}(\alpha)$.

PROOF. Pick probabilities $\left\{\lambda_{n}\right\}_{n=0}^{\infty}$ such that

$$
\sum_{n=0}^{\infty} \lambda_{n} \exp \left\{n \gamma \int_{[0, \infty)} x d F(x)\right\}=\infty
$$

(It is an elementary exercise to see that such probabilities exist.) Then

$$
\int_{[0, \infty)} e^{\gamma x} d H(x)=\sum_{n=0}^{\infty} \lambda_{n}\left(\int_{[0, \infty)} e^{\gamma x} d F(x)\right)^{n} \geq \sum_{n=0}^{\infty} \lambda_{n} \exp \left\{n \gamma \int_{[0, \infty)} x d F(x)\right\}
$$


is infinite by Jensen's inequality. Hence, we have $H \notin \mathcal{L}(\alpha)$ as $\int_{0}^{\infty} e^{\gamma x} d H(x)<\infty$ for $H \in \mathcal{L}(\alpha)$ and $\gamma<\alpha$ (see, for example, Cline [4, Lemma 2.2(i)]).

In Section 2 of this paper we explain what is wrong in the arguments of Cline [5]. In Section 3 we proceed to give a new proof of Cline's claimed results under the strengthened hypothesis that the probabilities $\left\{\lambda_{n}\right\}_{n=0}^{\infty}$ possess all exponential moments. This setting includes the important special case when $\left\{\lambda_{n}\right\}_{n=0}^{\infty}$ is a Poisson distribution (see, for example, Sato, [11, Section 25]), so that $H$ is a compound Poisson distribution with jump distribution in $\mathcal{L}(\alpha)$. In Section 4 we give an application in the form of a Tauberian criterion for infinitely divisible distributions to belong to $\mathcal{L}(\alpha)$.

As with the proof of Cline [4, Lemma 2.3(ii)], and half the proof of Cline [5], our arguments take off from the fundamental estimate (5) below from the proof of Embrechts and Goldie [6] that $\mathcal{L}(\alpha)$ is closed under convolution.

\section{The proofs by Cline [4] and [5]}

Let $F, G \in \mathcal{L}(\alpha)$. In order to show that $F \star G \in \mathcal{L}(\alpha)$, in equations (2.11) and (2.12) of the proof of their Theorem 3(b) Embrechts and Goldie [6] show that

$$
\frac{\overline{F \star G}(x-t)}{\overline{F \star G}(x)} \leq \max \left\{\sup _{y \geq x-v+t} \frac{\bar{F}(y-t)}{\bar{F}(y)}, \sup _{y \geq v} \frac{\bar{G}(y-t)}{\bar{G}(y)}\right\} \quad \text { for } x, v, t \in \mathbb{R} .
$$

It should be noted that although (seemingly) Embrechts and Goldie only claim (5) for $t>0$, an inspection of their proof shows that it holds in the above generality. The point of considering $t \in \mathbb{R}$, rather than $t>0$ only, is that (5) can then be used in the proof of both the lower bound (4) and the corresponding upper bound to get $H \in \mathcal{L}(\alpha)$, rather than in the proof of the lower bound only. This is crucial for our arguments, as the estimates Embrechts and Goldie develop for upper bounds in [6, p. 254] on seem to be too complicated for our application, as well as possibly insufficient.

In addition, Embrechts and Goldie [6] only consider distributions $F$ and $G$ supported on $(0, \infty)$ when establishing (5). However, we will use (5) for $F$ and $G$ supported on $[0, \infty)$, as the proof of Embrechts and Goldie carries over to this setting without any changes at all.

By iteration of (5) we obtain the following crucial lemma.

LEMMA 2.1. Let $F \in \mathcal{L}(\alpha)$ be supported on $[0, \infty)$ for some $\alpha \geq 0$. Given constants $\varepsilon>0$ and $t \in \mathbb{R}$, pick a constant $x_{0} \in \mathbb{R}$ such that (using (1))

$$
\frac{\bar{F}(x-t)}{\bar{F}(x)} \leq(1+\varepsilon) e^{\alpha t} \quad \text { for } x \geq x_{0} .
$$

Then

$$
\frac{\overline{F^{n \star}}(x-t)}{\overline{F^{n \star}}(x)} \leq(1+\varepsilon) e^{\alpha t} \quad \text { for } x \geq n\left(x_{0}-t\right)+t .
$$


Proof. As (6) gives (7) for $n=1$, it is enough to prove that, for any $k \geq 1$, (7) holds for $n=k+1$ if it holds for $n=k$. So assume that (7) holds for $n=k$, and take $G=F^{\star k}$ and $v=k x /(k+1)+t /(k+1)$ in (5). We have

$$
\begin{aligned}
\frac{\overline{F^{\star(k+1)}}(x-t)}{\overline{F^{\star(k+1)}}(x)} & \leq \max \left\{\sup _{y \geq x /(k+1)+k t /(k+1)} \frac{\bar{F}(y-t)}{\bar{F}(y)}, \sup _{y \geq k x /(k+1)+t /(k+1)} \frac{\overline{F^{\star k}}(y-t)}{\overline{F^{\star k}}(y)}\right\} \\
& \leq(1+\varepsilon) e^{\alpha t} \quad \text { for } x \geq(k+1)\left(x_{0}-t\right)+t,
\end{aligned}
$$

by (6) and (7) (with $n=k$ ), as requested, as

$$
\frac{x}{k+1}+\left.\frac{k t}{k+1}\right|_{x=(k+1)\left(x_{0}-t\right)+t}=x_{0}
$$

and

$$
\frac{k x}{k+1}+\left.\frac{t}{k+1}\right|_{x=(k+1)\left(x_{0}-t\right)+t}=k\left(x_{0}-t\right)+t .
$$

Now, as noted by Cline himself in [5], the problem with the proof of (4) in Cline [4, Lemma 2.3(ii)] is that (5) and (6) are used to make the incorrect deduction

$$
\frac{\overline{F^{n \star}}(x-t)}{\overline{F^{n \star}}(x)} \leq(1+\varepsilon) e^{\alpha t} \quad \text { for } x \geq x_{0}
$$

(see (7)), and that (8) (with its uniform bound for all $n$ ) is crucial for Cline's proof.

Moving on to the proof that $F \in \mathcal{L}(\alpha)$ implies $H=\sum_{n=0}^{\infty} \lambda_{n} F^{\star n} \in \mathcal{L}(\alpha)$ for $\alpha \geq 0$ in Cline [5] (recall Proposition 1.1), it makes crucial use of the inequality

$$
\overline{F^{\star n}}(x) \leq \bar{F}(x) \text { for } x>0 \text { (large enough) and } n \geq 1 .
$$

But the correct inequality here goes in the other direction, as (trivially)

$$
\overline{F^{\star n}}(x) \geq \overline{F^{\star m}}(x) \quad \text { for } x \in \mathbb{R} \text { and } n \geq m \geq 1 .
$$

In fact, it is well known that the limit in (3) is infinite for many members of $\mathcal{L}(\alpha)$ when $\alpha>0$, such as, for example, for an exponential distribution with expected value $1 / \alpha$, which combines with (10) to give

$$
\lim _{x \rightarrow \infty} \frac{\overline{F^{\star n}}(x)}{\bar{F}(x)} \geq \lim _{x \rightarrow \infty} \frac{\overline{F^{\star 2}}(x)}{\bar{F}(x)}=\infty \quad \text { for } n \geq 2 .
$$

That is, not only is (9) wrong, but rather the left-hand side can be (asymptotically) infinitely bigger that the right-hand side, which makes the proof of Cline [5] break down.

The result (4) of Cline [4] could still be correct for general probabilities $\left\{\lambda_{n}\right\}_{n=0}^{\infty}$, as Proposition 1.1 only shows that the corresponding upper bound (introduced in Cline [5]) is not correct in general. However, a proof of (4) is still missing. 


\section{A new proof of a version of the result of Cline [5]}

By Proposition 1.1, the result of Cline [5] that $\sum_{n=0}^{\infty} \lambda_{n} F^{\star n} \in \mathcal{L}(\alpha)$ for $F \in \mathcal{L}(\alpha)$ is incorrect in the absence of certain exponential moments for the probabilities $\left\{\lambda_{n}\right\}_{n=0}^{\infty}$. However, in the presence of such exponential moments we now give a new proof of this result.

TheOREM 3.1. Let $F \in \mathcal{L}(\alpha)$ be supported on $[0, \infty)$ for some $\alpha \geq 0$. Let the probabilities $\left\{\lambda_{n}\right\}_{n=0}^{\infty}$ satisfy $\lambda_{0}<1$ and $\sum_{n=0}^{\infty} \lambda_{n} e^{n \gamma}<\infty$ for each $\gamma>0$. We have $H=\sum_{n=0}^{\infty} \lambda_{n} F^{\star n} \in \mathcal{L}(\alpha)$.

ProOF. Picking $\varepsilon, t>0$, we have by ((1) and) Lemma 2.1,

$$
\frac{\overline{F^{\star n}}(x+t)}{\overline{F^{\star n}}(x)} \leq(1+\varepsilon) e^{-\alpha t} \text { for } x \geq n\left(x_{0}+t\right) \text {, for some } x_{0} \geq 0 .
$$

Let $c=1 /\left(x_{0}+t\right)$ and pick a constant $\gamma \in(\alpha / c, \infty)$. As $F \in \mathcal{L}(\alpha)$ implies that $\lim _{x \rightarrow \infty} e^{\beta x} \bar{F}(x)=\infty$ for $\beta>\alpha$ (see, for example, Cline [4, Lemma 2.2(i)]), we have $e^{-c \gamma x} \leq \varepsilon e^{-\alpha t} \bar{F}(x)$ for $x \geq x_{1}$, for some $x_{1} \geq x_{0}$. It follows that

$$
\begin{aligned}
& \sum_{n=N}^{\infty} \lambda_{n} \overline{F^{\star n}}(y) \\
& \quad \leq \sum_{n=N}^{\infty} \lambda_{n} \quad \text { (trivially) } \\
& \quad \leq e^{-\gamma N} \sum_{n=N}^{\infty} \lambda_{n} e^{\gamma n} \quad(\text { as } n \geq N) \\
& \leq e^{-c \gamma x} \quad\left(\text { as } N \geq c x \text { and } \sum_{n=N}^{\infty} \lambda_{n} e^{\gamma n} \leq 1 \text { for } N \text { large enough }\right) \\
& \quad \leq \varepsilon e^{-\alpha t} \bar{F}(x) \quad(\text { by initial construction }) \\
& \quad \leq \frac{\varepsilon}{1-\lambda_{0}} e^{-\alpha t} \sum_{n=1}^{\infty} \lambda_{n} \overline{F^{\star n}}(x) \quad\left(\text { by }(10) \text { and as } \sum_{n=1}^{\infty} \lambda_{n}=1-\lambda_{0}\right) \\
& \quad \leq \frac{\varepsilon}{1-\lambda_{0}} e^{-\alpha t} \bar{H}(x) \quad \text { for } y \in \mathbb{R}, N \geq c x \text { and } x \geq x_{2} \quad \text { (trivially) }
\end{aligned}
$$

for some $x_{2} \geq x_{1}$. On the other hand, (11) shows that

$$
\sum_{n=0}^{N-1} \lambda_{n} \overline{F^{\star n}}(x+t) \leq \sum_{n=0}^{N-1} \lambda_{n}(1+\varepsilon) e^{-\alpha t \overline{F^{\star n}}}(x) \leq(1+\varepsilon) e^{-\alpha t} \bar{H}(x),
$$

for $N \leq c x$ (so that $x \geq N\left(x_{0}+t\right)$ ) and $x \geq x_{0}$. Putting (12) and (13) together, we get

$$
\bar{H}(x+t)=\sum_{n=0}^{\infty} \lambda_{n} \overline{F^{\star n}}(x+t) \leq\left(1+\varepsilon+\frac{\varepsilon}{1-\lambda_{0}}\right) e^{-\alpha t} \bar{H}(x) \text { for } x \geq x_{2} .
$$


Turning to a lower bound, note that, picking constants $\varepsilon, t>0$, Lemma 2.1 readily gives

$$
\frac{\overline{F^{\star n}}(x+t)}{\overline{F^{\star n}}(x)} \geq(1-\varepsilon) e^{-\alpha t} \quad \text { for } x \geq n\left(x_{3}+t\right), \text { for some } x_{3} \geq 0 .
$$

Writing $c=1 /\left(x_{3}+t\right)$ and $N=\lfloor c x\rfloor+1$ and picking a constant $\gamma \in(\alpha / c, \infty)$, we therefore have

$$
\begin{aligned}
\bar{H} & (x+t) \\
& \geq \sum_{n=0}^{N-1} \lambda_{n} \overline{F^{\star n}}(x+t) \quad \text { (trivially) } \\
& \geq \sum_{n=0}^{N-1} \lambda_{n}(1-\varepsilon) e^{-\alpha t \overline{F^{\star n}}}(x) \quad\left(\text { as } n \leq N-1=\lfloor c x\rfloor \leq c x=x /\left(x_{3}+t\right)\right) \\
\geq & \sum_{n=0}^{\infty} \lambda_{n}(1-\varepsilon) e^{-\alpha t} \overline{F^{\star n}}(x)-\frac{\varepsilon}{1-\lambda_{0}} e^{-\alpha t} \bar{H}(x) \quad(\text { by }(12)) \\
& =\left(1-\varepsilon-\frac{\varepsilon}{1-\lambda_{0}}\right) e^{-\alpha t} \bar{H}(x) \quad \text { for } x \geq x_{2} \vee x_{3} \quad \text { (trivially). }
\end{aligned}
$$

Putting (14) and (15) together, we conclude that $H$ satisfies (1) for $t>0$, so that $H \in \mathcal{L}(\alpha)$, as is the claim of the theorem.

In the particular case when $F \in \mathcal{S}(\alpha)$ and $\left\{\lambda_{n}\right\}_{n=0}^{\infty}$ is a Poisson distribution, the conclusion of Theorem 3.1 follows from Embrechts and Goldie [7, Theorem 4.2(ii)].

\section{A Tauberian result for infinitely divisible distributions}

The following easy corollary to Theorem 3.1 is used in a crucial manner by Albin and Sundén [1].

Corollary 4.1. Let $G$ be an infinitely divisible distribution with Lévy measure $v$ such that $F(x)=v([1 \vee x, \infty)) / v([1, \infty)) \in \mathcal{L}(\alpha)$ for some $\alpha \geq 0$. Then $G \in \mathcal{L}(\alpha)$.

Proof. Writing $\left(\nu, m, \sigma^{2}\right)$ for the Lévy triplet of $G$, we have $G=F_{1} \star F_{2}$, where $F_{1}$ and $F_{2}$ are infinitely divisible distributions with Lévy triplets $(\nu([1, \infty)) d F, 0,0)$ and $\left(v(\cdot \cap(-\infty, 1)), m, \sigma^{2}\right)$, respectively. As $F_{1}$ is a compound Poisson distribution with jump distribution $F \in \mathcal{L}(\alpha)$ supported on $[1, \infty)$, Theorem 3.1 shows that $F_{1} \in \mathcal{L}(\alpha)$. Hence it follows from Pakes [9, Lemma 3.1] that $G=F_{1} \star F_{2} \in \mathcal{L}(\alpha)$.

In the particular case when $F \in \mathcal{S}$ the conclusion of Corollary 4.1 follows from Embrechts et al. [8, Theorem 1].

In the particular case when $F \in \mathcal{S}(\alpha)$, the conclusion of Corollary 4.1 follows from Braverman and Samorodnitsky [3, equation (3.37)]; see also Braverman 
[2, Proposition 1.3], and Pakes [9, Theorem 3.1]. However, as remarked by Braverman and Samorodnitsky [3, p. 229], the result for $F \in \mathcal{S}(\alpha)$ ' ... has been undoubtedly known to (among other people) Embrechts and Goldie, who included in their paper (1982) only the compound Poisson case...' (see the last paragraph of Section 3).

Added in Proof: The conclusion of Corollary 4.1 in the special case of the exponential class $\mathcal{S}(\alpha)$ may be found in [12].

\section{Acknowledgement}

We are grateful to Daren Cline for several helpful discussions, to Paul Embrechts for kind advice, and to a referee for helpful comments.

\section{References}

[1] J. M. P. Albin and M. Sundén, 'On the asymptotic behaviour of Lévy processes. Part I: subexponential and exponential processes', Preprint, 2007. http://www.math.chalmers.se/ palbin/levyI.pdf.

[2] M. Braverman, 'Suprema and sojourn times of Lévy processes with exponential tails', Stochastic Process. Appl. 68 (1997), 265-283.

[3] M. Braverman and G. Samorodnitsky, 'Functionals of infinitely divisible stochastic processes with exponential tails', Stochastic Process. Appl. 56 (1995), 207-231.

[4] D. B. H. Cline, 'Convolutions of distributions with exponential tails', J. Austral. Math. Soc. (Series A) 43 (1987), 347-365.

[5] - 'Convolutions of distributions with exponential tails: corrigendum', J. Austral. Math. Soc. (Series A) 48 (1990), 152-153.

[6] P. Embrechts and M. Goldie, 'On closure and factorization properties of subexponential and related distributions', J. Austral. Math. Soc. (Series A) 29 (1980), 243-256.

[7] _ 'On convolution tails', Stochastic Process. Appl. 13 (1982), 263-278.

[8] P. Embrechts, M. Goldie and N. Veraverbeke, 'Subexponentiality and infinite divisibility', Z. Wahrsch. 49 (1979), 335-347.

[9] A. G. Pakes, 'Convolution equivalence and infinite divisibility', J. Appl. Probab. 44 (2007), 295-305.

[10] E. J. G. Pitman, 'Subexponential distribution functions', J. Austral. Math. Soc. (Series A) 29 (1980), 337-347.

[11] K. Sato, Lévy processes and infinitely divisible distributions (Cambridge University Press, Cambridge, 1999).

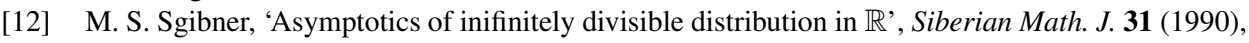
115-119.

[13] T. Shimura and T. Watanabe, 'Infinite divisibility and generalized subexponentiality', Bernoulli 11 (2005), 445-469.

\section{J. M. P. ALBIN, Department of Mathematics, Chalmers University of Technology, 41296 Gothenburg, Sweden e-mail: palbin@math.chalmers.se}

